\title{
Desorption by Ultrasound: Phenol on Activated Carbon and Polymeric Resin
}

\author{
Salil U. Rege and Ralph T. Yang \\ Dept. of Chemical Engineering, University of Michigan, Ann Arbor, MI 48109 \\ Charles A. Cain \\ Dept. of Biomedical Engineering, University of Michigan, Ann Arbor, MI 48109
}

\begin{abstract}
An experimental feasibility study of using ultrasound to accomplish the difficult desorption of phenol from activated carbon and polymeric resin adsorbents is discussed. The desorption rates of activated carbon were found to significantly increase by ultrasound at $40 \mathrm{kHz}$ and $1.44 \mathrm{MHz}$. Attrition of the activated carbon due to cavitation could be prevented by operating at a higher frequency and with an intensity below the threshold of the pulverization of carbon. According to the structural stability study of Amberlite XAD-4 and Dowex Optipore L-493 resins to withstand the abrasive cavitational effects of ultrasound at $40 \mathrm{kHz}$, the Dowex Optipore resin was stable under experimental conditions and phenol desorption rates were enhanced significantly with sonication. The ultrasonic desorption rates were favored by decreased temperature, aerated liquid medium, and increased ultrasound intensity. The desorption rates obtained without ultrasound appeared to be limited by pore diffusion, whereas those obtained in the presence of ultrasound were limited by surface reaction. The rate enhancement was due to an increase in diffusive transport within the pores caused by acoustic vortex microstreaming. The activation energy for desorption decreased with an increase in ultrasonic power density, thus making the ultrasound weaken the adsorption bond.
\end{abstract}

\section{Introduction}

One of the popular means for removal and recovery of organic water pollutants is by adsorption. Indeed, recently there has been an increasingly large amount of literature devoted to the study of adsorption for the removal of aqueous organic species such as phenols and substituted phenols using activated carbon (Grant and King, 1990; Stenzel, 1993; Karanfil et al., 1994; Leng and Pinto, 1996; Khan et al., 1997; Kilduff and King, 1997; Wang et al., 1997) and macrorecticular polymeric resins (Farrier et al., 1979; Goto et al., 1984; Furuya et al., 1989; Winkler et al., 1996; Lee and Ku, 1996). Although adsorption of phenolic compounds onto adsorbents such as granular activated carbon (GAC) and polymeric resins is relatively simple, the process of regenerating the adsorbent by desorption of the organic adsorbate still poses a major challenge to workers in this field, notably because of the high affinity of the compounds to the sorbent surface. The high initial cost of these adsorbents adds to the importance of the

Correspondence concerning this article should be addressed to R. T. Yang. problem of sorbent regeneration. The most common techniques for desorption of phenol from activated carbon are thermal regeneration (Suzuki et al., 1978; Moreno-Castilla et al., 1995; Torrents et al., 1997), chemical regeneration (Cooney et al., 1983; McLaughlin, 1995; Leng and Pinto, 1996; Kilduff and King, 1997; Rinkus et al., 1997), and to a smaller extent, regeneration under supercritical conditions (Modell et al., 1980; Recasens, 1989) and bioregeneration (Hutchinson and Robinson, 1990). The thermal regeneration method suffers from severe drawbacks, such as the requirement of high temperatures of the order of $300-500^{\circ} \mathrm{C}$ and excessive burnout of the carbon, resulting in capacity losses of 5-15\% (Grant and King, 1990), although efforts are now underway to lower the temperature (Torrents et al., 1997). Chemical regeneration, the other most common method, requires the use of organic solvents or inorganic chemicals and involves a secondary separation step, such as distillation, extraction, or chemical reaction. Supercritical regeneration of the adsorbent involves extracting the adsorbate into a supercritical 
fluid, such as $\mathrm{CO}_{2}$; however, although it has been shown to be highly successful in certain applications, high capital and operating costs have kept this technology from being commercially viable. Bioregeneration of the activated carbon has been studied using microbial degradation, but this method is highly time-consuming since most biological activities are slow. These techniques pose such problems that it is natural to explore other methods of desorption of adsorbates from the adsorbent surface. Other desorption techniques, such as application of electrical, magnetic, or acoustic fields, exist, but have not been explored so far. Of these, the application of an acoustic field, particularly in the form of an ultrasonic field, appears to be most promising for overcoming the high affinity of the adsorbates with adsorbent surface and their economical recovery.

In recent times, the study of ultrasonics and sonochemistry has expanded considerably and now finds diverse applications in industry, medical, as well as other fields, as can be inferred from various review books written on the subject (Suslick, 1988; Mason and Lorimer, 1988; Ensminger, 1988). A rapidly growing area is that of "environmental sonochemistry," which deals with destruction of organics in aqueous solution. This method aims at reduction of the polluting organics from aqueous waste streams by their chemical decomposition into simpler environmentally safe compounds (Hoffmann et al., 1996). However, the recovery of the organics in their original form, especially if they are valuable chemicals, would be more preferable than their chemical destruction.

Methods for the nondestructive recovery of organics from substrates using ultrasound have recently found an application in the study of decontamination of soil and sediments. Liu et al. (1996) and Mukherjee et al. (1997) have shown that sonication can significantly release organic matter adsorbed onto the particulate phase of aquatic sediments, thus increasing bioavailability of the sorbate. Similar studies have been conducted by Newman et al. (1997), with granular pieces of brick impregnated with copper oxide as a model for contaminated soil. They observed a $40 \%$ reduction in copper content by washing the model substrate by passing water on an ultrasonically shaken tray operating at $20 \mathrm{kHz}$.

Applications such as those mentioned earlier suggested that ultrasonically enhanced regeneration of adsorbents was a distinct possibility. However, little or no work in this regard has been reported in the literature, with the exception of that of Qin et al. (1997). This study, performed concurrently with our work, examined the desorption in NKA-II resin-TBP-50\% alcohol and CL-TBP resin-phenol systems and reported a shift in adsorption equilibrium in the presence of ultrasound. However, the authors neither discussed the possibility of degradation of their resin in ultrasound, nor explained the mechanism of desorption. Furthermore, only the change in adsorption equilibrium in the presence of ultrasound was discussed, whereas the desorption of phenol in pure water is of more practical importance.

The present work discusses the enhancement in desorption of phenol from activated carbon and polymeric resin in the presence of ultrasound. The stability of the sorbents under the action of ultrasonic cavitation is discussed in detail and influence of parameters such as frequency, power intensity, temperature, and degassing of the regenerant (water) are discussed. In addition, an attempt is made to explain the likely mechanism of ultrasonic desorption.

\section{Experimental Section}

\section{Activated carbon regeneration}

The activated carbon used in the phenol adsorption experiments was PCB $4 \times 10$ LS Calgon Activated Carbon (Calgon Carbon Corp., Pittsburgh). The carbon was pretreated by boiling in deionized water for $1 \mathrm{~h}$, followed by drying in the oven at $110^{\circ} \mathrm{C}$ overnight. Activated carbon $(0.8 \mathrm{~g})$ was added to $400 \mathrm{~mL}$ of $350 \mathrm{mg} / \mathrm{L}$ phenol solution prepared from stock solution of approximately $88 \%$ liquefied phenol (Mallinkodt AR). The activated carbon adsorbent was added to the solution in a sealed conical flask and placed on a shaker maintained at $20^{\circ} \mathrm{C}$ for three days. The carbon was then separated from the solution using vacuum filtration. The supernatant filtrate was analyzed by measuring its adsorbance using a DMS90 Varian UV-Visible Spectrophotometer at $270-\mathrm{nm}$ wavelength. The activated carbon that was now loaded with phenol was divided into two portions. One part was regenerated by adding $200 \mathrm{~mL}$ deionized water and placing the sample in an ultrasonic laboratory cleaner (Model T-14, L\&R Manufacturing Co., Kearney, NJ) operating at a frequency of $40 \mathrm{kHz}$ with an instrument power of $120 \mathrm{~W}$. The other part was regenerated by adding the same quantity $(200 \mathrm{~mL})$ of deionized water followed by stirring with a magnetic stirrer. In each case $10-\mathrm{mL}$ samples were withdrawn at regular time intervals, filtered to remove suspended impurities, and analyzed for phenol content spectrophotometrically.

The experiment at $40 \mathrm{kHz}$ revealed that considerable pulverization of the activated carbon occurred. It is known that at higher frequencies, the threshold intensity for cavitation increases since it becomes more difficult for creation of cavitation bubbles at low acoustic intensities (Mason and Lorimer, 1988). The experiment described earlier was then repeated using a high-intensity shallow-dish focused ultrasonic transducer operating at $1.44 \mathrm{MHz}$ with a total power of $100 \mathrm{~W}$. In a preliminary experiment, the intensity of the ultrasonic field was gradually increased until the threshold intensity for pulverization of the carbon particles was realized. The phenol regeneration experiments were conducted just below the pulverization threshold intensity. For the high-frequency experiments, two samples, each of $7 \mathrm{~g}$ of wetted activated carbon, were equilibrated with $100 \mathrm{~mL}$ of $3,820 \mathrm{mg} / \mathrm{L}$ of phenol solution as described before and then regenerated in $200 \mathrm{~mL}$ of deionized water. One sample was irradiated with ultrasound at $1.44 \mathrm{MHz}$, while the other sample was stirred using a magnetic stirrer. The sonication was administered in pulses of 2 $\mathrm{s}$, with a rest period of $2 \mathrm{~s}$ between two consecutive pulses in order to prevent overheating of the transducer. Hence, although the experiment was conducted for $30 \mathrm{~min}$, the actual sonication time was only $15 \mathrm{~min}$. Samples of $5 \mathrm{~mL}$ were withdrawn at regular time intervals for analysis using the UV-visible spectrophotometer.

\section{Resin stability in the presence of ultrasound}

Friable solids such as resin beads present in a liquid radiated with ultrasound are likely to be fractured and/or eroded because of the high-pressure shock waves resulting from cavity implosions (Suslick et al., 1987; Stephanis et al., 1997). Polymeric adsorbents usually have a macroreticular structure consisting of an agglomeration of a large number of very small microspheres fused together to form the resin bead. It was 
Table 1. Physical Properties of the Polymeric Resin Used in the Experiments

\begin{tabular}{lc}
\hline & Dowex Optipore Resin \\
\hline Description & Macroporous styrenic \\
& polymer \\
Bead size & $20-50$ mesh \\
Porosity $(\mathrm{v} / \mathrm{v})$ & 0.72 \\
Average pore diameter $(\AA)$ & 46 \\
Crush strength $(\mathrm{g} /$ bead $)$ & $>500$ \\
\hline
\end{tabular}

quite possible that due to the shock waves produced during ultrasonic cavitation the agglomerate would be fractured, resulting in severe particle-size reduction in due course of time. Hence a preliminary study of several types of polymeric resins was performed and the stability of the resin beads under the influence of an ultrasonic field was experimentally determined. Among the resin adsorbents studied, only two had the potential for separation of phenol from water and only these will be discussed in this work. One of the resins used was XAD-4/1090 Amberlite polymeric resin (Rohm and Haas Co.), which is a cross-linked polystyrene-type polymer. The other resin was Dowex Optipore L493 polymeric adsorbent (Dow Chemical Co.), which is a methylene bridged copolymer of styrene and divinylbenzene. The Dowex Optipore resin is also referred to as "tough gel," and is among the ones with the highest mechanical strengths. The resin samples were weighed before sonication, added to water, and then irradiated with ultrasound at $40 \mathrm{kHz}$ for $2 \mathrm{~h}$. After sonication the resin samples were filtered and dried. Size distribution was determined by sieving the samples through a battery of sieves, followed by weighing the size fractions. A similar procedure was followed in order to obtain the particle-size distribution before sonication. For the XAD-4 resin, sieves with Tyler equivalent mesh Nos. 32 and 48 were employed, and for the Optipore resin, mesh Nos. 28, 32, and 48 were used. The properties of the Dowex Optipore resin used are summarized in Table 1.

\section{Resin adsorption/desorption experiments}

On performing the resin stability experiments under the influence of ultrasound, it was found that the XAD-4 resin tended to be pulverized with time, whereas the Dowex Optipore resin was found to be stronger. Hence phenol adsorption/desorption experiments were performed only with the Optipore resin. The supplied resin beads were pretreated by washing with $200 \mathrm{~mL}$ water to remove traces of preservative agents and residual monomeric compounds, followed by a methanol wash, and finally again with deionized water. The resin was then vacuum filtered and placed in an oven at $120^{\circ} \mathrm{C}$ for $12 \mathrm{~h}$ for drying. Phenol solution $(200 \mathrm{~mL})$ of $400-\mathrm{mg} / \mathrm{L}$ concentration was prepared using deionized water and $6.2 \mathrm{~g}$ of pretreated dried resin was added to it. The resin-solution mixture was taken in a sealed conical flask and placed in a shaker maintained at $20^{\circ} \mathrm{C}$ for $24 \mathrm{~h}$. The phenol solution in the flask was analyzed for phenol content both before and after adsorption. The resin was then filtered from the phenol solution using vacuum filtration. Three samples of $4 \mathrm{~g}$ each of resin saturated with phenol were prepared. Two samples were taken in self-sealing polythene bags to be used for ultrasonic desorption while the third sample was taken in a conical flask to be desorbed by stirring. The use of polythene bags was intended to match the acoustic impedance of the two media, thus lowering the degree of attenuation of acoustic power, and hence increasing the acoustic intensity incident on the resin sample. Degassed deionized water $(150 \mathrm{~mL})$ was prepared by boiling for $10 \mathrm{~min}$ and subsequently cooling it in a sealed flask to $20^{\circ} \mathrm{C}$. Deionized water $(150 \mathrm{~mL})$ was added to a sample in one of the polythene bags, while an equivalent volume of degassed deionized water was added to the other polythene bag and sealed. The two resin samples were separately immersed in the ultrasonic laboratory cleaner bath containing water, as described earlier, and were subject to ultrasound at $40 \mathrm{kHz}$. Preliminary experiments had shown that there was a rise in temperature of the liquid in the ultrasonic bath of the order of $5-10^{\circ} \mathrm{C}$, primarily because of the heating up of the transducers. In order to maintain the temperature of the ultrasonic bath constant, the water in the bath was circulated continuously using a constant temperature bath (Neslab Endocal refrigerated circulating bath) maintained at $20^{\circ} \mathrm{C}$. Temperature of the solution containing the adsorbent was monitored at regular time intervals and was found to be constant within $\pm 1^{\circ} \mathrm{C}$. During the course of ultrasonic treatment of the sample, samples of $5 \mathrm{~mL}$ each were withdrawn at fixed time intervals from the polythene bags for analysis of phenol content using a UV-spectrophotometer. Deionized water $(150 \mathrm{~mL})$ was added to the sample in the conical flask and the phenol from the resin was desorbed using stirring caused by a magnetic stirrer. As described before, samples of $5 \mathrm{~mL}$ were withdrawn from the solution and analyzed. The experiment described earlier was repeated at $57^{\circ} \mathrm{C}$ in order to determine the effect of temperature on ultrasonic desorption. It must be noted, however, that the adsorption of phenol was done under identical conditions as those existing for the experiment at $20^{\circ} \mathrm{C}$. Degassed water, however, was not used for the experiment at $57^{\circ} \mathrm{C}$, as was done for that at $20^{\circ} \mathrm{C}$. The regenerating water samples were first heated to $60^{\circ} \mathrm{C}$, and then added to the resin adsorbent saturated with phenol. The constant temperature circulation bath was employed as before to maintain the required temperature. In order to study the effect of power intensity on the desorption rate, the voltage to the ultrasonic bath was reduced to $50 \%$ of peak power (i.e., $60-\mathrm{W}$ power) using a rheostat, and the experiments discussed before were repeated at this reduced power at $20^{\circ} \mathrm{C}$ and $52^{\circ} \mathrm{C}$.

\section{Results and Discussion}

\section{Regeneration of activated carbon}

The concentration of phenol in the deionized water regenerating the activated carbon was monitored with time; both in the presence of ultrasound of $40-\mathrm{kHz}$ frequency, and without ultrasound but with stirring. The results of the desorption experiments are shown in Figure 1. It shows that the rate of phenol desorption was significantly increased in the presence of ultrasound. The rate of desorption by stirring of the carbon slurry was found to be almost negligible. It is known that phenol undergoes irreversible oxidative coupling on carbon surfaces, forming high molecular-weight phenol dimers and trimers under conditions similar to the ones in our adsorption experiments (Grant and King, 1990), although we have 
not verified this fact in our work. These oligomers are difficult to desorb, which might explain the low desorption rates obtained by simple stirring of the carbon slurry in water. The generation of "hot spots" due to cavitation and acoustic microstreaming in the pores seemed to enable the rupture of the strong sorbate-surface bonds and improve desorption rate when an ultrasonic field was applied. It must be noted that the UV-spectrometric technique used to measure the amounts of sorbate desorbed in the regenerant solution could only measure phenol in the monomeric form reliably at $270 \mathrm{~nm}$. The phenol dimers or trimers, if at all formed on the surface and subsequently desorbed, would not be detected, although it is known that a phenol dimer (dihydroxybiphenyl or biphenol) does adsorb at a wavelength of $255 \mathrm{~nm}$-close to the $270-\mathrm{nm}$ wavelength used in our measurements. Hence, there is a possibility that the curves shown in the figure may be an underestimate of the actual amount desorbed ultrasonically.

It can be noticed from Figure 1 that the last data point in the ultrasonic desorption curve strayed upwards from the preceding trend. Unfortunately, this experiment was not repeated to verify this trend for certain technical reasons. However, as will be seen in the subsequent sections on the desorption of phenol from polymeric resin, it is a common feature of the data obtained in the presence of ultrasound to move upwards from the initial trend toward the end of desorption, and hence the last data point in Figure 1 does not appear to be in error. This anomaly, as will be explained in detail later on, can be attributed to a shift from pore-diffusion regime to surface-reaction-controlled regime toward the end of the desorption process due to depletion of surface sorbate concentration, as well as due to the high energetic heterogeneity on the sorbent surface.

It was also observed that the activated carbon was progressively eroded due to the shock waves generated by isolated imploding cavities and subsequent creation of powerful liquid jets when the cavities were created near the surface. As a result the solution was found to gradually accumulate large

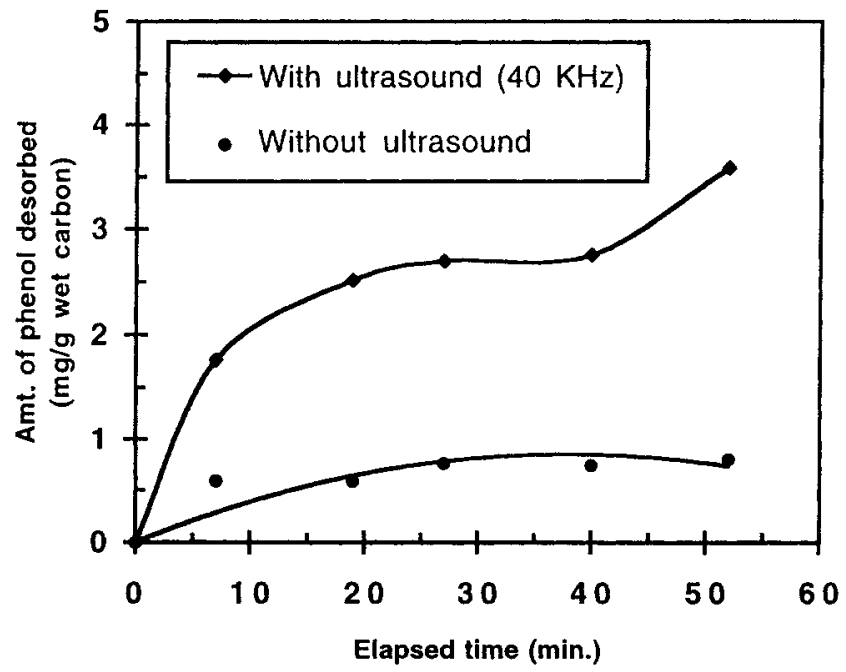

Figure 1. Amount of phenol desorbed from Calgon PCB activated carbon in deionized water per weight of wet carbon vs. time at $20^{\circ} \mathrm{C}$ using ultrasound at $40 \mathrm{kHz}(120-\mathrm{W}$ power) and by stirring without ultrasound. quantities of colloidal carbon particles with exposure to the low-frequency ultrasound. However, the amount of particle reduction was not quantified in this work. This destruction of the activated carbon particles was unacceptable for practical purposes, and in order to avoid this problem, it was necessary to reduce the effect of cavitation. It is known that at high ultrasonic frequencies, the rarefaction and compression wave cycles are too short to permit the cavitating bubble to grow to a size sufficient to cause disruption in liquid (Mason and Lorimer, 1988), thus decreasing the production and intensity of cavitation in liquids at high frequencies. Hence the ultrasonic desorption experiment was conducted at a higher frequency of $1.44 \mathrm{mHz}$, as discussed in the experimental section. The transducer used afforded flexibility in adjustment of acoustic intensity, and by proper manipulation of the sonic intensity, destruction of the activated carbon was reduced considerably by operating just below the threshold for carbon pulverization. Change in the frequency of sound simply alters the resonant size of cavitation bubble and there is no direct coupling of the sound field with the adsorbed species on the molecular level (Ley and Low, 1989). Hence the nature of desorption curve was not expected to be altered significantly at high frequencies.

The results of the phenol desorption in the presence of ultrasound in comparison with the desorption rate obtained without ultrasound but with stirring of the activated carbon are shown in Figure 2. As was discussed in the experimental section, the actual time of sonication was half of the experiment time since the pulses had rest periods of equal duration in between. It was seen that there was an enhancement in desorption of phenol at $1.44 \mathrm{MHz}$, but to a smaller extent compared to that at $40 \mathrm{kHz}$. Since this particular experiment was conducted at an intensity just below the pulverizing threshold of carbon, the amount of cavitation and hence extent of desorption would be lower.

There has been literature on the degradation of organic contaminants in water by ultrasound that reported the decomposition of the phenolic compounds to form corresponding oxidized products in the region of $20 \mathrm{kHz}$ (Hoffmann et

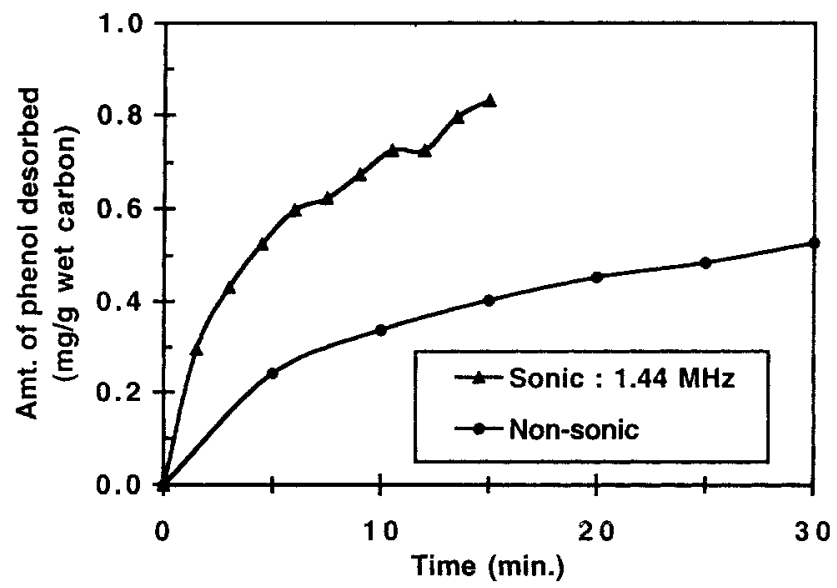

Figure 2. Amount of phenol desorbed from Calgon PCB activated carbon in deionized water per weight of wet carbon vs. time at $20^{\circ} \mathrm{C}$ using ultrasound at $1.44 \mathrm{MHz}$ (100-W power) and by stirring without ultrasound. 
al., 1996) and $0.8 \mathrm{MHz}$ (Ensminger, 1988) under high-power ultrasound of about $80 \mathrm{~W}$. However, our experiments with a blank phenol sample of $32 \mathrm{mg} / \mathrm{L}$ phenol solution revealed no detectable destruction of phenol at both $40 \mathrm{kHz}$ and $1.44 \mathrm{MHz}$ under the conditions of the experiment.

\section{Stability of polymeric resins under ultrasonic field}

The other type of adsorbents that is popularly used for removal of organics from aqueous waste streams is the polymer adsorbents. Since the experiments with activated carbon revealed that ultrasound had a destructive effect on friable solids, preliminary experiments were conducted to determine if the polymeric resin beads could withstand the implosive shock waves created due to cavitation at $40 \mathrm{kHz}$. Figure 3 shows the size distribution of XAD-4 resin, while Figure 4 shows that of the Dowex Optipore resin before and after ultrasonic irradiation of $40 \mathrm{kHz}$ over a period of $2 \mathrm{~h}$. It was observed that the XAD-4 resin underwent a size reduction, as is apparent from the increase in percentage of particles retained on Tyler Mesh No. 48 at the cost of decrease in particles retained on Tyler Mesh No. 32. Also the solution appeared to become turbid after $30 \mathrm{~min}$ of ultrasonic irradiation, thus indicating that cavitation was causing the macroreticular structure of the resin beads to be fractured. However, in the case of the Optipore resin (or, the "tough gel"), no such size reduction was observed. The size distribution appeared to retain its characteristics after ultrasonic exposure, notably because of its superior crush strength. The apparent increase in the percentage of the particles retained on Tyler Mesh No. 32 was probably caused due to swelling of resin particles lying initially in the range of Tyler Mesh No. 48. Hence the Dowex Optipore resin was found suitable for ultrasonic desorption experiments.

\section{Regeneration of polymer resin}

Dowex Optipore resin beads were saturated with phenol at $20^{\circ} \mathrm{C}$ and were subjected to ultrasonic desorption as described before. The desorption of organics from a porous adsorbent can be explained by different effects of ultrasound in heterogeneous media. First, "hot spots" are created within the liquid where the temperature and pressure of the gas in the cavity rises to enormously high values due to expansion and implosive collapse of bubbles at nucleation sites within the liquid irradiated with ultrasound. Creation of high temperature can obviously enhance the breaking of physical and chemical bonds between the adsorbate and the adsorbent surface. Although the breaking of hydrogen bonds in the desorption of phenol from resin was attributed by Qin et al. (1997) to this "spot energy effect," no specific reasoning was provided in their work. Second, acoustic cavitation produces high-speed microjets and high-pressure shock waves that impinge on the surface and erode the adsorbate (Suslick et al., 1987; Stephanis et al., 1997). Finally, ultrasound produces acoustic vortex microstreaming within porous solids as well as at the solid-liquid interface. This phenomenon is caused due to the increase in momentum brought about as the solvent adsorbs energy from the propagating sound wave and is found to occur even in the absence of cavitation (Ley and Low, 1989). Such an effect was found to accelerate mass transport in large-pore membranes (Arkhangel'skii and Statnikov,

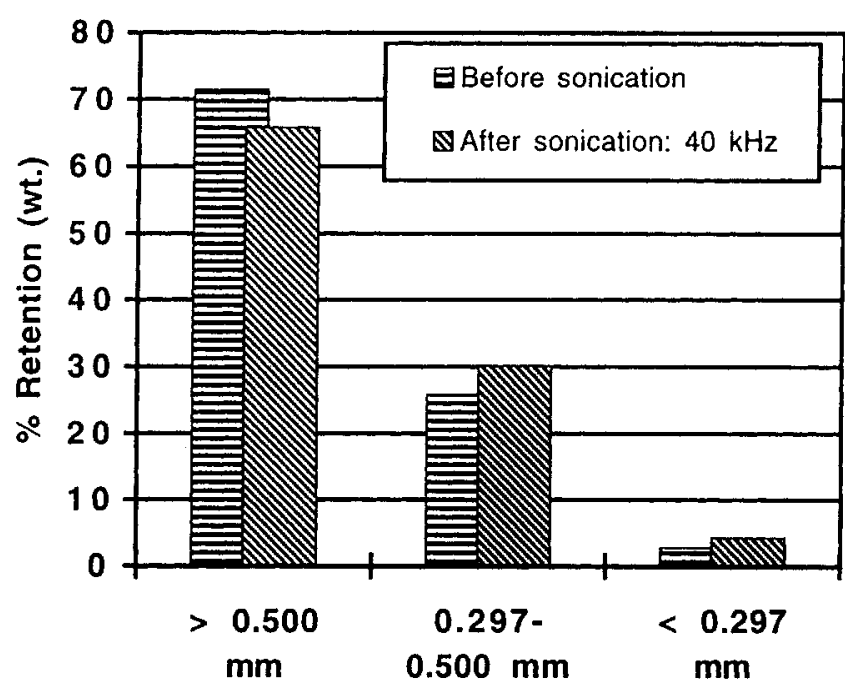

Figure 3. Size distribution of XAD-4 resin obtained by sieving with Tyler Nos. $32(0.500-\mathrm{mm})$ and 48 $(0.297-\mathrm{mm})$ mesh sizes before and after irradiation with ultrasound at $40 \mathrm{kHz}(120 \mathrm{~W})$ for $2 \mathrm{~h}$.

1973), and could possibly be the cause of enhanced desorption rates. In the present study, an effort was made to determine which of the effects was important for the desorption of organics from polymeric resin adsorbents.

\section{Desorption at $20^{\circ} \mathrm{C}$}

The Dowex Optipore resin that was saturated with phenol adsorbate as discussed earlier, was subjected to ultrasonic irradiation at $40 \mathrm{kHz}$. The results of the desorption experiment with ultrasound using deionized water as well as degassed deionized water as a regenerant at $20^{\circ} \mathrm{C}$ are shown in Figure

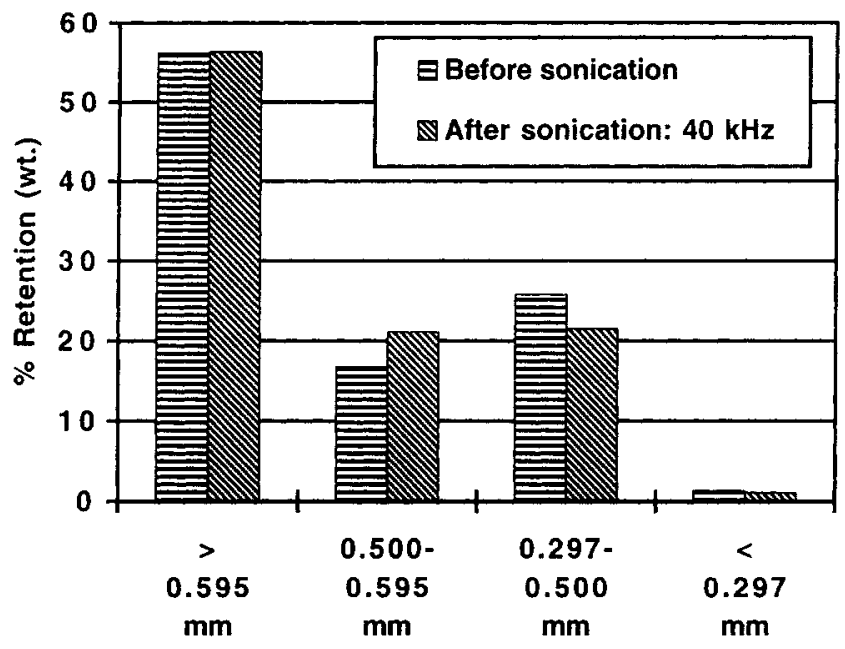

Figure 4. Size distribution of Dowex Optipore L493 resin obtained by sieving with Tyler Nos. 28 $(0.595-\mathrm{mm}), 32(0.500-\mathrm{mm})$, and 48 $(0.297-\mathrm{mm})$ meshes before and after irradiation with ultrasound at $40 \mathrm{kHz}(120 \mathrm{~W})$ for $2 \mathrm{~h}$. 


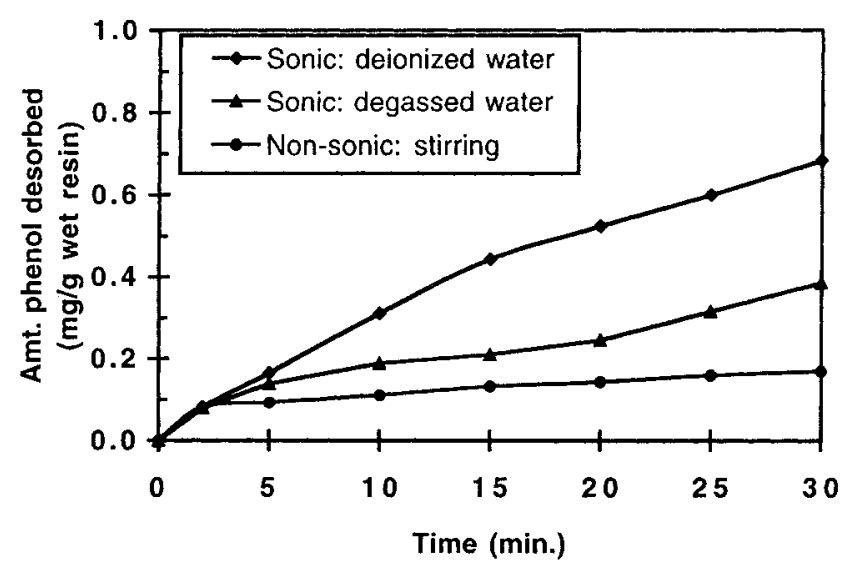

Figure 5. Amount of phenol desorbed per weight of wet resin vs. time for phenol desorption from Dowex Optipore resin at $20^{\circ} \mathrm{C}$ using ultrasound at $40 \mathrm{kHz}(120 \mathrm{~W})$ with aerated deionized water and degassed deionized water.

Lower desorption curve shows the profile obtained by stirring the resin without ultrasound.

5. The amount of phenol desorbed per weight of wet resin obtained by the stirring of the solution at $20^{\circ} \mathrm{C}$ is also shown for comparison. There seems to be a "jump" in the concentration of phenol just after the start of the desorption experiment, which is not consistent with the rest of the desorption curve. This jump was probably caused by the diffusion of phenol retained within the macropores of the resin bead out into the bulk phase. Since this amount of phenol was not adsorbed in the microspheres, and since diffusion from the macropores of the resin was a fast process, there was a sudden, small increase in phenol content in the bulk regenerant phase. From the figure it is obvious that the rate of phenol desorption is much higher in the presence of ultrasound than is obtained by simple stirring of the resin in the regenerating solution. It should be noted that the present experiment was performed using a laboratory ultrasonic cleaner that had little flexibility with regards to intensity of ultrasonic radiation as well as its frequency. Hence the results obtained may not necessarily be the optimum results using ultrasound as a means of regenerating the spent adsorbent.

\section{Effect of degassed water as regenerant}

To understand the role of cavitation in the rate of desorption of the adsorbate from the resin, the ultrasonic experiment was also conducted using degassed water as a regenerant. The degassed water was prepared by boiling deionized water for $10 \mathrm{~min}$ and subsequently cooling it to $20^{\circ} \mathrm{C}$. The residual gas content in the water following the degassing procedure was not measurable, but it has been shown that a considerable amount of gases may be retained in liquids, even after the degassing procedure (Entezari and Kruus, 1996). Degassing the liquid substantially lowers the number of nucleating sites available for microbubbles to grow and cavitate. Thus, the cavitating threshold intensity is increased, and hence the effect due to cavitation is substantially lowered. Figure 5 shows the desorption curve obtained when a de- gassed water solution was used instead of normal (aerated) water in the presence of ultrasound. It can be seen that the phenol desorption rate was higher than that obtained without sound, but it was substantially lower than that obtained for aerated solution. This showed that cavitation played a significant role in ultrasonic desorption. The rate of desorption was still higher than ordinary diffusion induced by stirring, which proved that either a small amount of cavitation was still acting (due to the incomplete degassing of water or the creation of nucleating sites by the porous resins) or the residual enhancement was due to acoustic microstreaming within the pores of the resin particle. The latter effect occurs even in the absence of cavitation. The lower rate of reaction in the case of degassed solutions is a commonly observed phenomenon in sonochemical literature (e.g., Mason and Lorimer, 1988; Ley and Low, 1989; Entezari and Kruus, 1996).

\section{Effect of temperature of the regenerating solution}

The desorption of phenol from the resin surface could be either limited by the breaking of strong surface bonds or by the diffusion of the phenol molecules through the micropores of the resin. In order to study the influence of either limiting steps, an estimate of the kinetic parameters was required. Hence the phenol desorption experiments were repeated at $57^{\circ} \mathrm{C}$. The results of the experiments are shown in Figure 6. From the figure, it can be seen that the rates of desorption, in general, were much higher than the corresponding rates observed at $20^{\circ} \mathrm{C}$. This was expected since desorption is an activated process. It was interesting to note, however, that the enhancement in desorption rate due to ultrasound over that by stirring was negligibly small compared to that observed at $20^{\circ} \mathrm{C}$. In general, cavitating bubbles are more easily produced as the temperature is increased because of the decrease in surface tension and viscosity of the liquid medium. However, there is also a dramatic increase in the vapor pressure of the liquid. Thus cavitating bubbles at high temperatures of the bulk liquid tend to have greater vapor content, which lowers the effect of cavitational collapse. The maximum temperature and pressure created in such vaporous

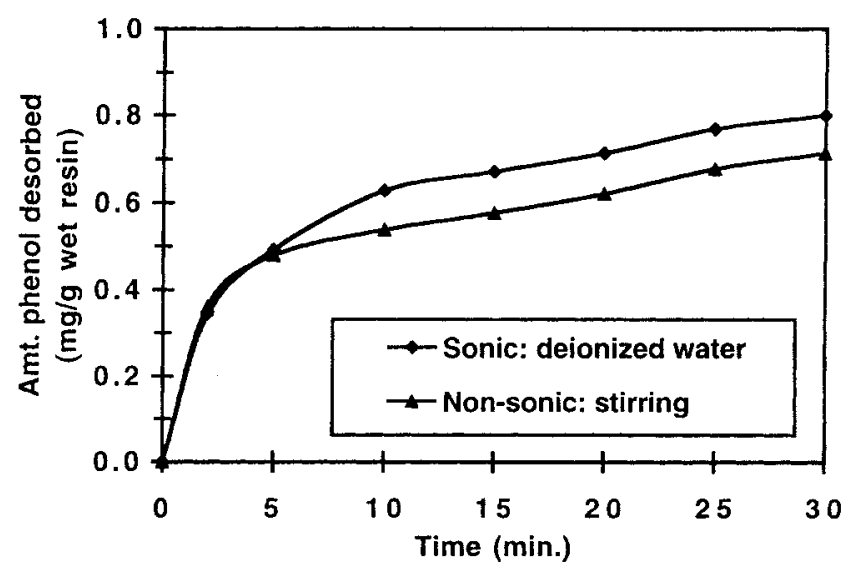

Figure 6. Amount of phenol desorbed per weight of wet resin vs. time for the desorption of phenol from Dowex Optipore resin at $57^{\circ} \mathrm{C}$ using ultrasound at $40 \mathrm{kHz}$ with deionized water and by stirring the resin without ultrasound. 
transient cavitating bubbles have been shown to be much lower (Mason and Lorimer, 1988). Besides, while heating the regenerating water to $57^{\circ} \mathrm{C}$, substantial degassing of the water occurred, which might also explain the low enhancement in desorption rate. Thus, the observed behavior of phenol desorption at higher temperature is consistent with the present theoretical understanding of sonochemistry.

\section{Effect of ultrasonic power}

The ultrasonic cleaner was operated at $40 \mathrm{kHz}$ and at a total instrument power of $120 \mathrm{~W}$. To study the influence of the power density of ultrasound on the desorption rate, the power to the instrument was reduced to $50 \%$ of the peak value by using a powerstat. The experiment of phenol desorption was studied at the reduced power of $60 \mathrm{~W}$ again at two temperatures, namely $20^{\circ} \mathrm{C}$ and $55^{\circ} \mathrm{C}$. The results of the experiment are shown in Figure 7. From the figure, it can be seen that the rates of desorption are much reduced in comparison to those obtained using $100 \%$ power. Compared to the rates of desorption obtained by stirring, as seen from the lower curves of Figures 5 and 6 at $20^{\circ} \mathrm{C}$ and $57^{\circ} \mathrm{C}$, respectively, the rates of desorption at $60 \mathrm{~W}$ are only marginally higher. Thus, it was concluded that the degree of ultrasonic power plays an important role in enhancing desorption.

\section{Desorption models, kinetic rate constants, and mechanism of ultrasound desorption}

In order to gain a better understanding of the mechanism and to quantify the rates of desorption obtained in the resin desorption experiments, it was necessary to propose a suitable model for the desorption. Various mechanisms have been suggested in the literature to explain the adsorption-desorption kinetics in resins. Furuya et al. (1989) concluded that the pore-diffusion model fitted their data best for the phenol/XAD-2000 resin system. On the other hand, Lee and $\mathrm{Ku}$ (1996) showed that the ion-exchange surface reaction was dominant in the kinetics of chlorophenol/Purolite A.510

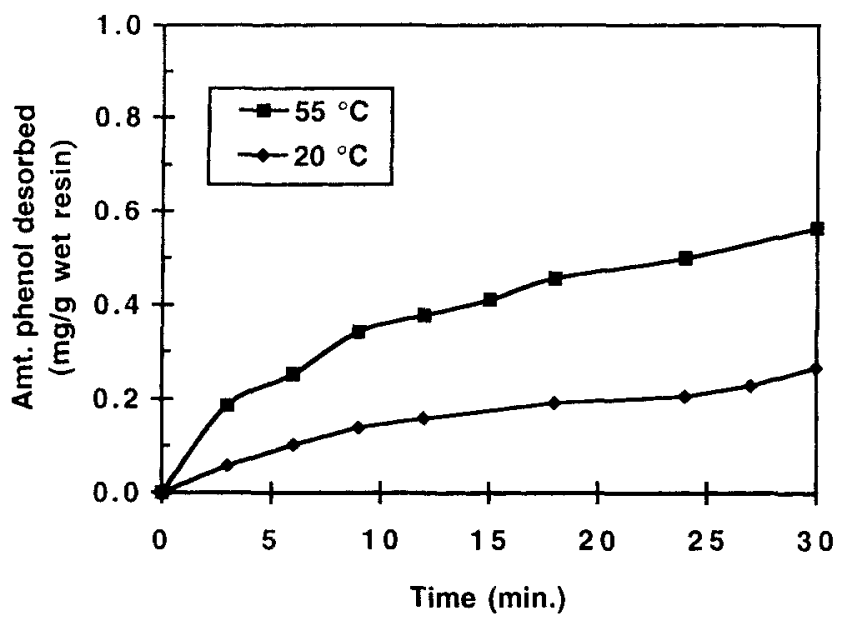

Figure 7. Amount of phenol desorbed per weight of wet resin vs. time for the desorption of phenol from Dowex Optipore resin at $20^{\circ} \mathrm{C}$ and $55^{\circ} \mathrm{C}$ using ultrasound at $40 \mathrm{kHz}$ and reduced power of $60 \mathrm{~W}$ with deionized water. resin. Both the resins quoted here were macroreticular polystyrene divinylbenzene resins. In the present work, both the models, namely those of pore diffusion and surface kinetics, were considered and compared against experimental data for the goodness of fit.

The average pore diameter of the Optipore resin as given in Table 1 is $46 \AA$. Since the least molecular diameter of a phenol molecule is $2.5 \AA$, the kinetics could possibly be pore-diffusion controlled. For isothermal diffusion in a spherical adsorbent particle of radius, $r$, the sorption curve has been shown to follow the following equation (Crank, 1979):

$$
\frac{M_{t}}{M_{\infty}}=1-\frac{6}{\pi^{2}} \sum_{n=1}^{\infty} \frac{1}{n^{2}} \exp \left(-\frac{D}{r^{2}} n^{2} \pi^{2} t\right)
$$

One of the assumptions of this equation is that the surface concentration of the spherical particle is constant. Hence this equation was fit using the initial data obtained immediately after the start of the desorption experiment, that is, when the concentration of the regenerating solution remained essentially unchanged. In the short time region, Eq. 1 approaches the limiting parabolic form:

$$
\frac{M_{t}}{M_{\infty}}=6 \sqrt{\frac{D t}{r^{2} \pi}}
$$

From the desorption data obtained from the experiments described before, the fractional amount desorbed $\left(M_{t} / M_{\infty}\right)$ was calculated at various times. By plotting $M_{t} / M_{\propto}$ vs. $\sqrt{t}$, values for the diffusivity rate constants $\left(D / r^{2}\right)$ were calculated from the slope, and are given in Table 2 .

It was also possible that the desorption rate was limited by the breaking of the surface bonds that bound the adsorbate molecule with the sorbent surface. Intuitively the rate of desorption was proposed to be a first-order reaction process:

$$
-\frac{d C}{d t}=k_{d} C
$$

Table 2. Diffusivity Constants, Assuming the Pore Diffusion Model, Surface Reaction Rate Constants, and First-Order Kinetics*

\begin{tabular}{ccc}
\hline & $\begin{array}{c}\text { Diffusivity Constant, } \\
D / r^{2}\left(\mathrm{~s}^{-1}\right)\end{array}$ & $\begin{array}{c}\text { First-Order Rate } \\
\text { Constants }\left(\mathrm{s}^{-1}\right)\end{array}$ \\
\hline $\begin{array}{ccc}\text { Without ultrasound } \\
T=20^{\circ} \mathrm{C}\end{array}$ & $2.5 \times 10^{-6}$ & $1.6 \times 10^{-4}$ \\
$T=57^{\circ} \mathrm{C}$ & $8.8 \times 10^{-5}$ & $1.2 \times 10^{-3}$ \\
With ultrasound $(60 \mathrm{~W})$ & & \\
$T=20^{\circ} \mathrm{C}$ & $4.6 \times 10^{-6}$ & $2.2 \times 10^{-4}$ \\
$T=55^{\circ} \mathrm{C}$ & $2.5 \times 10^{-5}$ & $7.2 \times 10^{-4}$ \\
With ultrasound $(120 \mathrm{~W})$ & & \\
$T=20^{\circ} \mathrm{C}$ & $3.0 \times 10^{-5}$ & $9.2 \times 10^{-4}$ \\
$T=20^{\circ} \mathrm{C}$ & $7.8 \times 10^{-6}$ & $3.3 \times 10^{-4}$ \\
degassed sample & & \\
$T=57^{\circ} \mathrm{C}$ & $1.1 \times 10^{-4}$ & $2.0 \times 10^{-3}$ \\
\hline
\end{tabular}

*For the desorption of phenol from Dowex Optipore resin with ultrasound at $40 \mathrm{kHz}$ and with stirring without sound. 
where $C=$ concentration of adsorbate (phenol) on the adsorbent surface (mg/g wet resin) at time $t$, and $k_{d}=$ desorption rate constant $\left(\mathrm{s}^{-1}\right)$.

Integrating Eq. 3 gives

$$
\ln \left(\frac{C}{C_{o}}\right)=-k_{d} \cdot t,
$$

where $C_{o}=$ initial concentration of the phenol adsorbed on the adsorbent surface $\left(\mathrm{mg} / \mathrm{g}\right.$ wet resin). A plot of $\ln \left(C / C_{o}\right)$ vs. $t$ yielded a straight line equal to the desorption rate constant. The values of the first-order desorption kinetic rate constants are also summarized in Table 2.

In order to discriminate between the diffusion and the first-order surface kinetic models, the predictions of both were compared against the experimental fractional desorption data. Figure 8 shows the comparison of models for the experiments done at $20^{\circ} \mathrm{C}$, which corresponds with Figure 5 discussed earlier. It can be seen from the figure that although the pore diffusion model fitted the data fairly well for the desorption conducted without ultrasound, it failed to fit the data for desorption assisted with ultrasound. On the other hand, the first-order kinetic model showed excellent agreement with the ultrasonic desorption data, but its prediction of desorption in the absence of sound was not as good as that predicted by the pore-diffusion model. For the case of desorption in a degassed medium, both models were equally capable of fitting the data. Figure 9 shows a similar comparison for the experiments done at $57^{\circ} \mathrm{C}$ and corresponds with Figure 6 discussed earlier. Again, in this case the pore-diffusion model represented the data for nonsonic desorption more accurately than the first-order surface kinetic model, while the opposite seemed to be true for the sonic-desorption case. The

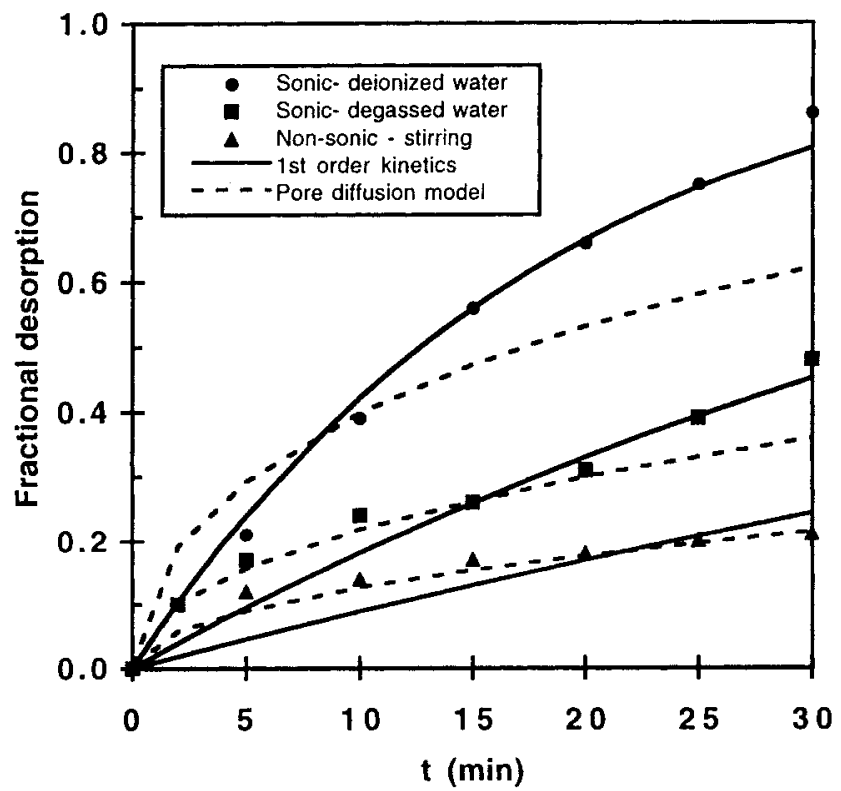

Figure 8. Fractional desorption of phenol from Dowex Optipore resin vs. time: comparison of first-order surface-kinetic model and pore-diffusion model with experimental data at $20^{\circ} \mathrm{C}$, corresponding to Figure 5.

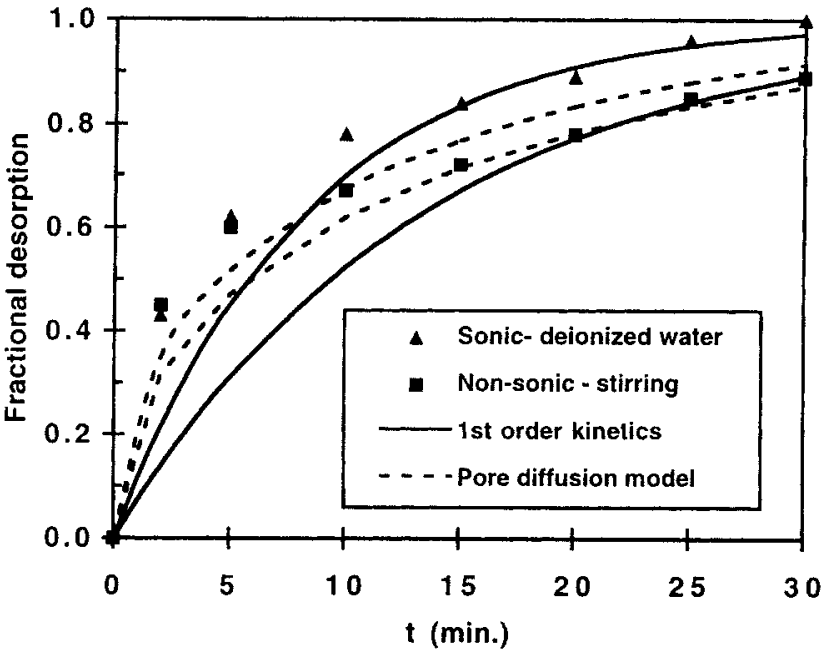

Figure 9. Fractional desorption of phenol from Dowex Optipore resin vs. time: comparison of first-order surface-kinetic model and pore-diffusion model with experimental data at $57^{\circ} \mathrm{C}$, corresponding to Figure 6 .

model predictions along with the experimental observations for the experiments done at $50 \%$ power (i.e., $60-\mathrm{W}$ instrument power) and at temperatures of $20^{\circ} \mathrm{C}$ and $55^{\circ} \mathrm{C}$ are shown in Figure 10 . At $20^{\circ} \mathrm{C}$, the pore-diffusion model was better than the kinetic model; but at $55^{\circ} \mathrm{C}$, although the rate data showed a propensity toward the diffusion model initially at longer times, it wandered toward the surface kinetic model.

A study of Figures 8-10 thus shows that pore-diffusion resistance was probably more dominant than surface reaction for desorption without ultrasound. For ultrasonic desorption, surface kinetics seemed to play a more significant role than pore diffusion. Although the model discrimination was by vi-

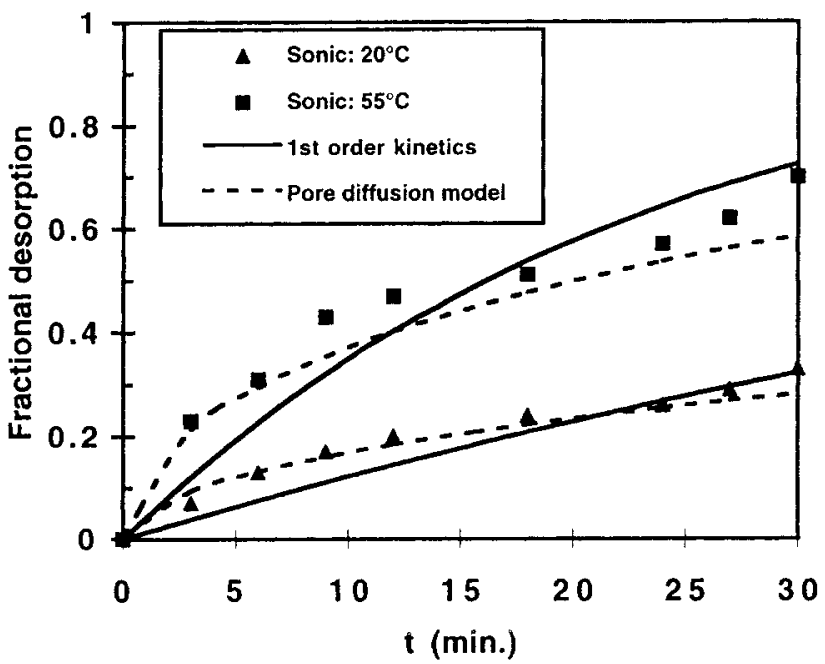

Figure 10. Fractional desorption of phenol from Dowex Optipore resin vs. time: comparison of first-order surface-kinetic model and pore-diffusion model with experimental data at $20^{\circ} \mathrm{C}$ and $55^{\circ} \mathrm{C}$ with $50 \%(60 \mathrm{~W})$ of the peak power, corresponding to Figure 7. 
sual judgment and admittedly subjective, it can be concluded with some confidence that ultrasonic desorption for the phenol-resin system mainly occurred due to enhancement of diffusion rates within the particle. It is known that on applying a sound field to a fluid medium within a pore or a channel, acoustic vortex microstreaming results. The increased microturbulence within the resin pores results in an increase in the rate of mass transport, thus enhancing the desorption rate in the presence of ultrasonic power. As was discussed earlier in Figure 5, the use of degassed water for regenerating the resin caused a lower desorption rate than was possible when water equilibrated with ambient air was used. This is now evident given the fact that degassing of the solution causes reduced cavitation and hence the amount of vortex microstreaming would be consequently decreased. Thus the diffusivity of the desorbed species is lowered due to degassing, yielding lower desorption rates.

Another interesting feature of the ultrasonic desorption curves is that the initial time data seems to be consistent with the pore-diffusion model, while that at longer times is in better agreement with the surface kinetic model. This is particiularly conspicuous in the degassed medium curve in Figure 8, the sonic desorption curves of Figures 9 and 10, and also in Figure 1 for the activated carbon regeneration which showed slightly sigmoidal curves bending upward toward the end of each experiment. The desorption appears to be diffusion limited initially, but with time, there is a shift to the reactioncontrolled regime. Initially, there is a large surface concentration of the adsorbate on the sorbent surface. As a result, the reaction rate is fast at the beginning since it is proportional to the surface concentration. Toward the end of the desorption, the surface concentration drops and hence the surface kinetic rate slows down. As a result there is a shift from initial pore-diffusion control to reaction control as time progresses. Furthermore, adsorbents with random pore structures such as the macroreticular resins and activated carbon are known to have a highly energetically heterogeneous surface. Thus some sorbate molecules are held strongly to the sorbent surface, whereas others are loosely bound. The initial time data obtained during ultrasound-assisted experiments thus represented the desorption of loosely held molecules, which were not limited by the breaking of surface bonds, but had substantial diffusion resistance. On the other hand, the data obtained at longer times corresponded to the tightly bound sorbate molecules on the surface, and obviously surface kinetics played a significant role in desorption of these molecules.

\section{Influence of ultrasound on the energy of activation}

The first-order kinetic rate constants calculated at two temperatures from the desorption curves (as in Table 2) obtained from the ultrasonic experiments at $50 \%(60-\mathrm{W})$ and $100 \%(120-\mathrm{W})$ power were fit to the Arrhenius equation with an objective of computing the energy of activation $\left(E_{\text {act }}\right)$ for the surface reaction in both the cases. The results of the calculation showed that the $E_{\text {act }}$ was about $7.5 \mathrm{kcal} / \mathrm{mol}$ at $50 \%$ of the peak power. When $100 \%$ of the available power $(120$ W) to the instrument was used, the $E_{\text {act }}$ dropped to 4.6 $\mathrm{kcal} / \mathrm{mol}$. Since the desorption caused by stirring without sound was not surface-reaction dependent, its energy of acti- vation could not be compared with that obtained in the presence of ultrasound. Nevertheless, as a matter of theoretical interest, the first-order rate constants for desorption without sound in Table 2 were assumed to hold, and the $E_{\text {act }}$ was calculated to be $11.9 \mathrm{kcal} / \mathrm{mol}$. This value is interestingly close to the heat of adsorption of phenol on macroreticular resin, which is known to be $12.6 \mathrm{kcal} / \mathrm{mol}$ (Farrier et al., 1978). Thus, it is apparent that the application of ultrasonic energy at different degrees of intensity causes a corresponding lowering of the energy of activation. Since it is known that ultrasound did not interfere with the adsorbate species on the molecular level in the experiments performed, as was proven by experiments with blank phenol samples, sonication could not have affected the reaction pathway. Hence, the lowering of energy of activation in the presence of ultrasonic energy can only be explained by the fact that a part of the activation energy was being substituted by the acoustic cavitation energy. As the amount of acoustic energy supplied was increased, the share of activation energy provided by the cavitation effect also increased.

\section{Conclusions}

In this work the feasibility for the use of ultrasound for desorption of phenol from activated carbon and polymeric resin was demonstrated to be encouraging. Both types of sorbents showed an enhancement in desorption rate in the presence of ultrasound at $40 \mathrm{kHz}$ that was greater than that observed in the nonsonic case. However, activated carbon showed a tendency to be pulverized under the action of ultrasound at $40 \mathrm{kHz}$. At a higher frequency of $1.44 \mathrm{MHz}$, the pulverization of carbon was prevented and a sufficient enhancement in desorption rate was obtained. The ultrasonic desorption method was particularly successful in the case of the polymeric resin adsorbent. The rates of desorption obtained by irradiating the adsorbent saturated with phenol using degassed water as a regenerant showed a considerably lower enhancement than was obtained with aerated water. This showed that acoustic cavitation played an important role in the desorption process. Experiments carried out at a higher temperature of $57^{\circ} \mathrm{C}$ showed that although the rates of desorption were in general higher than those obtained at $20^{\circ} \mathrm{C}$, the enhancement was much lower since cavitation was of a predominantly transient vaporous nature. The ultrasonic method for desorption was found to be favored by low temperature, by the use of an aerated solution, and by an increase in power intensity. In order to understand the mechanism of ultrasonic desorption, pore diffusion and first-order surface kinetic models were proposed and corresponding rate constants were obtained. It was found that desorption of phenol from the resin sorbent was pore-diffusion limited, whereas ultrasonic desorption was limited by the surface reaction. Thus, enhancement in desorption rates in the presence of ultrasound can be attributed to an enhancement in diffusional transport due to the acoustic microstreaming caused within the pores. The energy of activation $\left(E_{\text {act }}\right)$ calculated by assuming the desorption being a first-order process, was found to decrease with an increase in the degree of ultrasonic power. This proved that the acoustic cavitation energy increasingly provides the $E_{\text {act }}$ for desorption as power intensity is increased. 
The results in this work were obtained using phenol as a model compound, but they should also apply to other organic sorbates on similar sorbents. Thus, the ultrasonic method for the recovery of adsorbates may well be a convenient alternative to chemical/thermal regeneration methods, particularly when the adsorbate species is chemically unreactive or is thermally sensitive. However, further work is necessary with regards to the optimization of intensity and frequency of ultrasonic radiation to be applied, as well as the development of new adsorbents with greater mechanical strength to withstand the abrasive action of cavitation for the successful implementation of this process.

\section{Acknowledgments}

This work was supported by the NSF under Grant CTS-9520328. The authors are grateful to Jan-Ulco A. Kluiwstra of the Biomedical Engineering Department for his help in performing the experiments with high-frequency ultrasonic apparatus. They also thank an anonymous reviewer for helpful comments. The resin samples were kindly donated by Dow and Rohm \& Haas Companies.

\section{Notation}

$D=$ diffusivity of sorbate within the sorbent pores, $\mathrm{m}^{2} / \mathrm{s}$

$M_{T}=$ amount of sorbate desorbed from the sorbent surface at time $t$, $(\mathrm{mg} / \mathrm{g}$ wet resin)

$M_{x}=$ total amount of sorbate adsorbed on the sorbent surface, $(\mathrm{mg} / \mathrm{g}$ wet resin)

$n=$ summation variable in diffusion model

\section{Literature Cited}

Arkhangel'skii, M. E., and Yu. G. Statnikov, Physical Principles of Ultrasonic Technology, Vol. 2, L. D. Rozenberg, ed., Plenum Press, New York, p. 294 (1973).

Cooney, D. O., A. Nagerl, and A. L. Hines, "Solvent Regeneration of Activated Carbon," Water Res., 17, 403 (1983).

Crank, J., The Mathematics of Diffusion, 2nd ed., Clarendon Press, Oxford, p. 91 (1979).

Ensminger, D., Ultrasonics: Fundamentals, Technology, Applications, 2nd ed., Dekker, New York, p. 405 (1988).

Entezari, M. H., and P. Kruus, "Effect of Frequency on Sonochemical Reactions: II. Temperature and Intensity Effects," Ultrasonics Sonochem., 3, 19 (1996).

Farrier, D. S., A. L. Hines, and S. W. Wang, "Adsorption of Phenol and Benzoic Acid from Dilute Aqueous Solution onto a Macroreticular Resin," J. Colloid Interface Sci., 69, 233 (1979).

Furuya, E., Y. Takeuchi, and K. E. Noll, "Intraparticle Diffusion of Phenols Within Bidispersed Macroreticular Resin Particles," J. Chem. Eng. Jpn., 22, 670 (1989).

Goto, S., M. Goto, and S. Uchiyama, "Adsorption Equilibria of Phenol on Anion Exchange Resins in Aqueous Solution," J. Chem. Eng. Jpn., 17, 204 (1984).

Grant, T. M., and C. J. King, "Mechanism of Irreversible Adsorption of Phenolic Compounds by Activated Carbons," Ind. Eng. Chem. Res., 29, 264 (1990).

Hoffmann, M. R., I. Hua, and R. Höchemer, "Applications of Ultrasonic Irradiation for the Degradation of Chemical Contaminants in Water," Ultrasonics Sonochem., 3, S163 (1996).

Hutchinson, D. H., and C. W. Robinson, "A Microbial Regeneration Process for Granular Activated Carbon," Water Res., 24, 1209 (1996).

Karanfil, T., M. A. Schlautman, and W. J. Weber, Jr., "Impacts of Dissolved Oxygen on the Sorption of Humic Substances and the Subsequent Inhibition of O-Cresol Uptake by Granular Activated Carbon," Water Res., 28, 1673 (1994).
Khan, A. R., T. A. Al-Bahri, and A. Al-Haddad, "Adsorption of Phenol Based Organic Pollutants on Activated Carbon from MultiComponent Dilute Aqueous Solutions," Water Res., 31, 2102 (1997).

Kilduff, J. E., and C. J. King, "Effect of Carbon Adsorbent Surface Properties on the Uptake and Solvent Regeneration of Phenol," Ind. Eng. Chem. Res., 36, 1603 (1997).

Lee, K.-C., and Y. Ku, "Removal of Chlorophenols from Aqueous Solution by Anion-Exchange Resins," Sep. Sci. Technol., 31, 2557 (1996).

Leng, C.-C., and N. G. Pinto, "An Investigation of the Mechanisms of Chemical Regeneration of Activated Carbon," Ind. Eng. Chem. Res., 35, 2024 (1996).

Ley, S. V., and C. M. R. Low, Ultrasound in Synthesis, Chap. 2, Springer-Verlag, Berlin (1989).

Liu, D., I. Aoyama, H. Okamura, and B. J. Dutka, "Enhancement of Toxicant Release from Sediment by Sonication and Sodium Ligninsulfonate," Environ. Toxicol. Water Qual., 11, 195 (1996).

Mason, T. J., and J. P. Lorimer, Sonochemistry: Theory, Applications and Uses of Ultrasound in Chemistry, Chap. 2, Halsted Press (Wiley), Chichester, U.K. (1988).

McLaughlin, H. S., "Regenerate Activated Carbon Using Organic Solvents," Chem. Eng. Prog., 91, 45 (1995).

Modell, M., R. deFilippi, and V. Krukonis, "Regeneration of Activated Carbon with Supercritical Carbon Dioxide," Activated Carbon Adsorption of Organics from the Aqueous Phase, I. H. Suffet and M. J. McGuire, eds., Ann Arbor Science, Ann Arbor, MI (1980).

Moreno-Castilla, C., J. Rivera-Utrilla, J. P. Joly, M. V. Lopez-Ramon, M. A. Ferro-Garcia, and F. Carrasco-Marin, "Thermal Regeneration of Activated Carbon Exhausted with Different Substituted Phenols," Carbon, 33, 1417 (1995).

Mukherjee, S., R. Mathew, and H.-N. Hsieh, "Enhancing Bioavailability in Aquatic Sediments Using Ultrasound," Geotech. Spec. Publ., Vol. 65, ASCE, New York, p. 122 (1997).

Newman, A. P., J. P. Lorimer, T. J. Mason, and K. R. Hutt, "An Investigation into the Ultrasonic Treatment of Polluted Solids," $\mathrm{Ul}$ trasonics Sonochem., 4, 153 (1997).

Oin, W., Y. Yuan, and Y. Dai, "Studies on the 'Spot Energy Effect' of Ultrasound," Proc. China/USA Chem. Eng. Conf., Vol. II, Chem. Ind. Eng. Soc. (China), Beijing, p. 666 (1997).

Recasens, F., B. J. McCoy, and J. M. Smith, "Desorption Processes: Supercritical Fluid Regeneration of Activated Carbon," AIChE J., 35, 951 (1989).

Rinkus, K., B. E. Reed, and W. Lin, "NaOH Regeneration of $\mathrm{Pb}$ and Phenol-Laden Activated Carbon. I. Batch Study Results," Sep. Sci. Technol., 32, 2367 (1997).

Stenzel, M. H., "Remove Organics by Activated Carbon Adsorption," Chem. Eng. Prog., 89, 36 (1993).

Stephanis, C. G., J. G. Hatirisand, and D. E. Mourmouras, "The Process (Mechanism) of Erosion of Soluble Brittle Materials Caused by Cavitation," Ultrasonics Sonochem., 4, 269 (1997).

Suslick, K. S., Ultrasound: Its Chemical, Physical and Biological Effects, Chap. 5, VCH, New York (1988).

Suslick, K. S., D. J. Casadonte, M. L. H. Green, and M. E. Thompson, "Effects of High Intensity Ultrasound on Inorganic Solids," Ultrasonics, 25, 56 (1987).

Suzuki, M., D. M. Misic, O. Koyama, and K. Kawazoe, "Study of Thermal Regeneration of Spent Activated Carbons: Thermogravimetric Measurement of Various Single Component Organics Loaded on Activated Carbons," Chem. Eng. Sci., 33, 271 (1978).

Torrents, A., R. Damera, and O. J. Hao, "Low-Temperature Thermal Desorption of Aromatic Compounds from Activated Carbon," J. Hazard Mat., 54, 141 (1997).

Wang, R.-C., C.-C. Kuo, and C.-C. Shyu, "Adsorption of Phenols onto Granular Activated Carbon in a Liquid-Solid Fluidized Bed," J. Chem. Tech. Biotech., 68, 187 (1997).

Winkler, K., K.-H. Radeke, and H. Stach, "Adsorption/Desorption Behavior of Organic Water Pollutants onto an Adsorber Polymer," Chem. Tech. (Leipzig), 48, 249 (1996).

Manuscript received Jan. 23, 1998, and revision received Mar. 23, 1998. 